\title{
Strategies to Support the Integration of Behavioral Health and Primary Care: What Have We Learned Thus Far?
}

\author{
W. Perry Dickinson, MD
}

The articles in this supplement contain a wealth of practical information regarding the integration of behavioral health and primary care. This type of integration effort is complex and greatly benefits from support from outside organizations, as well as collaboration with other practices attempting similar work. This editorial extracts from these articles some of the key lessons learned regarding the integration of behavioral health and primary care for practices and for organizations that support practice transformation. (J Am Board Fam Med 2015;28:S102-S106.)

Keywords: Behavioral Medicine; Delivery of Health Care, Integrated; Mental Health; Primary Health Care; Quality of Health Care

The integration of behavioral health care and primary care has become an area of focus in health care redesign efforts, with evidence that integrated care is essential to accomplishing the Triple Aim. ${ }^{1-4}$ As in the other articles in this issue, we use the following definitions modified from Peek $^{5}$ and Butler and colleagues. ${ }^{6}$ Behavioral health care includes care for patients around mental health and substance use conditions, health behavior change, life stressors and crises, and stress-related physical symptoms. Integrated care is care rendered by a practice team of primary care and behavioral health providers, working together with patients and families and using a systematic and cost-effective approach to provide patient-centered care.

Although many primary care practices, including those involved in the studies described in this issue, have worked hard to implement integrated behavioral health care despite payment and other

This article was peer reviewed.

Submitted 30 March 2015; revised 26 April 2015; accepted 30 April 2015.

From the Department of Family Medicine, University of Colorado School of Medicine, Aurora.

Funding: This research was supported by a grant from the Colorado Health Foundation (CHF-3848).

Conflict of interest: none declared.

Corresponding author: Perry Dickinson, MD, Department of Family Medicine, University of Colorado School of Medicine, 12631 East 17th Avenue, Mail Stop F496, Aurora, CO 80045 (E-mail: perry.dickinson@ucdenver.edu). barriers, this involves trailblazing challenging terrain. The studies in this issue describe hard-earned lessons learned and effective strategies to assist practices embarking on this important work.

Practice transformation in general and behavioral health integration specifically are challenging innovations for practices that are bombarded with new programs requiring multiple major changes. Most primary care practices have few mechanisms for rapidly incorporating new programs, which can slow adoption of innovations and cause disruptions when innovations are finally implemented. ${ }^{7-11}$ Multiple methods have emerged to assist practices in practice transformation efforts. In particular, practice facilitation has emerged as a key method for assisting practices with organizational changes. ${ }^{7-8,12-15}$ Facilitators assist practices in implementing quality improvement and change management programs, improving incorporation of innovations into operations and increasing sustainability. Studies suggest that the use of practice facilitation and other methods such as learning collaboratives to support practices in difficult transformation and quality improvement efforts results in better outcomes. ${ }^{12-15}$ However, there are few studies that have specifically dealt with transformation support for behavioral health integration. What are the key lessons learned in this set of studies that can inform efforts to support practices in behavioral 
health integration? And what may be different for behavioral health integration efforts from other types of practice transformation? This editorial addresses those questions, with a primary focus on behavioral health integration in primary care practices.

\section{Team-Based Care and Change Management}

A particularly crucial area of focus for behavioral health integration is the establishment of highly functional interprofessional teams for patient care. Although this is important in most models for practice transformation, behavioral health integration centers on bringing together professionals who have traditionally operated with different approaches and philosophies for seamless, teambased care. This requires focused, intentional work that can be greatly assisted by outside facilitation. Many practices attempting behavioral health integration have just added people working in parallel in their own siloes and using their standard approaches to care. Although this can have limited success, it does not result in the advances and improvements in care that are possible through spending the time to develop new, team-based integrated approaches. Building on the different cultures and approaches brought by medical and behavioral clinicians, practices must develop a shared vision and mental model for integration to guide the transformation process. This involves changes and the development of new skills for everyone in the practice. ${ }^{16}$ An interprofessional, team-based process for implementing behavioral health integration helps in developing trust and rapport, learning each other's strengths and roles, understanding potential gaps, and developing effective work flows and care processes. Although the use of interprofessional quality improvement teams is standard for most practice transformation support efforts, it is particularly crucial for behavioral health integration.

\section{Workforce and Training Issues}

Practices implementing behavioral health integration models report considerable difficulty finding behavioral health clinicians trained in brief, solution-focused interventions, adaptable to the pace of primary care, and having an understanding of the broad-based needs of a primary care population. ${ }^{17}$ Practices also report challenges in finding primary care clinicians with a willingness and ability to work as a team, sharing the responsibility for patient care with other clinicians and incorporating their expertise into patient care. Primary care clinicians have to be able to identify and initially deal with patients' behavioral needs and then know when and how to involve behavioral health clinicians. Practice staff members require new skills in identifying behavioral health issues, facilitating screening and warm handoffs between clinicians, and dealing with sensitive and emotional issues that they may not have previously faced. All this requires thoughtful attention to training and mentoring of existing and new clinicians and staff members, with everyone learning together how to optimally adopt the new teambased integrated care. Practices that have been successful in adopting integrated models over time devote major time and effort to train and onboard new behavioral health and primary care clinicians. The onboarding process includes socializing the new clinicians to the practice culture, vision for integrated care, model of care and workflow, and promoting the development of trust and rapport and an understanding of everyone's skills and roles. This involves training programs, training manuals, an initial process of having clinicians shadow each other, ongoing mentoring, and ongoing interprofessional team meetings. ${ }^{17}$ Support organizations must collect best practices, assist in the development of shared training programs and resources, and assist in the development of mentoring across practices within a learning community.

\section{Care Processes and Structures}

Team-based care in behavioral health integration requires the careful consideration of how clinicians and staff will work together to meet the needs of specific patients. Cohen et $\mathrm{a}^{18}$ describe the use of consulting, coordinating, and collaborating as approaches depending on the clinical task. Huddles or other structured or unstructured meetings can be used to plan care on an ongoing basis, with interprofessional complex care meetings to focus on patients with more difficult issues. The ability to quickly access other clinicians to discuss patient issues or do warm handoffs often shapes the level of integration in the practice. ${ }^{18-19}$

Proximity of clinicians and staff members shapes integrated care through the logistic ease of making contact with each other. This requires a thoughtful 
shaping or adaptation of space, workflows, protocols, and data systems (electronic health records [EHRs] and shared care plans). As described in the Article by Gunn et al, ${ }^{19}$ the design of the physical space often shaped the quality and frequency of interactions and the level of integration of care. There is a necessary and important balance of proximity to encourage and enhance interaction and provision of personal workspace to perform work tasks with privacy and/or peace and quiet when needed. ${ }^{19}$ The use of cell phones, instant messaging, and other methods for asynchronous communication can help, but they are no substitute for physical proximity. Although extensive consultation regarding the design of space is likely beyond the capacity of most practice support organizations, it is important that these issues be raised for consideration for practices implementing integrated models of care.

Quality improvement teams implementing behavioral health integration must develop workflows and care pathways that are understood by everyone in the practice and that consider all aspects of the identification of behavioral health issues, the transitions of care between primary care and behavioral health clinicians, and the coordinated referral of patients needing more intensive or long-term care. Decisions regarding the identification of patients with behavioral health issues are a critical and deceptively complicated initial process needing consideration. Systematic screening results in a reliable process that identifies patients needing services and also provides data to better understand the needs of the patient population and to refine the integration approach. ${ }^{16}$ Systematic screening results in a greater reach of services and more accurate patient identification than nonsystematic screening that relies on clinician judgment. However, decisions regarding screening have to balance the targeted reach and the capacity of the available behavioral health services-identifying the targeted population, but being able to then deliver services to the identified patients. ${ }^{16,20-21}$ Broad-based, systematic screening can identify a large volume of behavioral health needs that can very quickly overwhelm the capacity of the available behavioral health clinicians. This requires careful planning and consideration of the different models for implementing behavioral health care in the practice. The consideration and tracking of reach to plan and fine tune interventions such as behavioral health integration is a fundamental skill for practices, and one that practice support organizations must begin to foster. $^{21}$

As is well described in the Article by Davis et $\mathrm{al},{ }^{20}$ the planning of patient scheduling and patient transitions is inescapably intertwined with decisions regarding patient identification. Although integrated behavioral health care models promote brief, primary care, problem-focused interventions and warm handoffs between clinicians, most behavioral health clinicians are trained in and more comfortable with traditional behavioral health interventions, with longer appointments with patients referred by the primary care clinician. For practices using more integrated approaches, the scheduling pattern can either enable or prevent real-time behavioral health clinician availability for warm handoffs and brief, integrated interventions. A variety of models are used for scheduling counseling visits while keeping availability for warm handoffs and dealing with issues emerging during care sessions. Scheduling return patients in a way that maintains availability for arising issues requires matching the pattern to the usual flow of patients in the practice, including identifying when real-time availability is most needed. The establishment of norms with staff and patients that interruptions are acceptable and permitted even while the behavioral health clinician is in the room with a patient also helps maintain availability. ${ }^{20}$

Scheduling behavioral health clinicians in traditional blocks for more intensive, traditional counseling has advantages in billing and predictable scheduling, with little or no down time for the clinicians. However, this model results in limited ability for warm handoffs, consultations, or brief interventions and predominantly works in a referral framework. Some practices are adopting combined models, with scheduling of behavioral health clinicians that can allow both longer visits for traditional counseling and strategic open slots for availability for warm handoffs and brief visits, or with different behavioral health clinicians employing different scheduling approaches to make room for both models of care.

Developing coordinated systems for consultation with or referral to psychiatry and long-term counseling resources is a crucial part of implementing integrated care. ${ }^{16}$ This can be challenging when the practice hires a behavioral health clinician directly instead of contracting for the clinician's ser- 
vices through a mental health center. This will often involve enhanced connections with a mental health center, community psychiatrists, and/or other community resources. Practice transformation support organizations can play an important role in facilitating connections among practices and community resources to improve the coordination of care in these situations.

\section{Data and Health Information Technology Issues}

EHRs have offered the promise of improved sharing of records across clinicians and the extraction of important clinical data for quality improvement and population management. However, there is perhaps no clinical area in which EHRs have fallen as short on this promise as in integrated care. ${ }^{22}$ Practices commonly use separate record systems for behavioral health and medical care, and even those working from the same EHR often restrict access to important behavioral health or primary care information due to HIPAA concerns. This results in clinicians having to use double documentation, scanning, and paper documents to work around these limitations. EHRs generally lack places to document behavioral health information as structured data that could be extracted from the system. All this results in difficulty for clinicians in communicating and sharing key information regarding patients, an inability to effectively track the care that patients are receiving, and a deficit of data for quality improvement regarding integration efforts. The sharing of patient information is crucial for team-based care, and data are necessary for the provision of effective care. Practice transformation organizations or other groups supporting practices in behavioral health integration efforts should include data support tailored to the practice circumstances among the services provided to practices.

\section{Conclusion}

This is an exciting era for primary care practice transformation, and no area of care is riper for innovation and change than behavioral health integration in primary care. This is not a one-size-fitsall phenomenon, as is clear from the reports in this issue. New and adaptive care models are needed to fit with local needs, practice context, and new payment models. This is a complex matching process with a tremendous amount of room for innovation.
Practices can be successful innovating on their own, but are generally much more successful working on complex issues such as behavioral health integration in learning collaboratives and with outside assistance. Practices and practice support organizations must learn from each other to move forward with behavioral health integration. The lessons learned that are shared in this FABFM supplement provide direction and a great start for such efforts.

\section{References}

1. Butler M, Kane RL, McAlpin D, et al. Integration of mental health/substance abuse and primary care No. 173 (Prepared by the Minnesota Evidence-based Practice Center under Contract No. 290-02-0009.) AHRQ Publication No. 09-E003. Rockville, MD: Agency for Healthcare Research and Quality, October 2008 .

2. Kwan B, Nease D Jr. The state of the evidence for integrated behavioral health in primary care. In: Talen MR, Burke Valeras A, eds. Integrated behavioral health in primary care. New York: Springer, 2013;65-98.

3. Katon W, Unützer J, Fan MY, et al. Cost-effectiveness and net benefit of enhanced treatment of depression for older adults with diabetes and depression. Diabetes Care 2006;29:265-70.

4. Unützer J, Katon WJ, Fan MY, et al. Long-term cost effects of collaborative care for late-life depression. Am J of Manag Care 2008;14:95-100.

5. Peek CJ. Lexicon for behavioral health and primary care integration: Concepts and definitions developed by expert consensus. Rockville, MD: Agency for Healthcare Research and Quality, 2013. Available from: http://integrationacademy.ahrq.gov/sites/ default/files/Lexicon.pdf. Accessed April 26, 2015.

6. Butler M, Kane RL, McAlpine D, et al. Integration of mental health/substance abuse and primary care. Evidence report/technology assessment No. 173. Rockville, MD: Agency for Healthcare Research and Quality, 2008. Available from: http://www.ahrq.gov/ research/findings/evidence-based-reports/mhsapcevidence-report.pdf. Accessed April 26, 2016.

7. Greco PJ, Eisenberg JM. Changing physicians' practices. N Engl J Med 1993;329:1271-73.

8. Nagykaldi Z, Mold JW, Aspy CB. Practice facilitators: A review of the literature. Fam Med 2005;37(8): 581-8.

9. Solberg LI, Brekke ML, Fazio ML, et al. Lessons from experienced guideline implementers: Attend to many factors and use multiple strategies. Jt Comm J Qual Improv 2000;26(4):171-88.

10. Tallia AF, Stange KC, McDaniel RR Jr, Aita VA, Miller WL, Crabtree BF. Understanding organiza- 
tional designs of primary care practices. Jt Comm J Qual Improv 2003;48(1):45-59; discussion 60-1.

11. Wagner EH, Austin BT, Davis C, Hindmarsh M, Schaefer J, Bonomi A. Improving chronic illness care: Translating evidence into action. Health Aff (Millwood). 2001;20(6):64-78.

12. Baskerville NB, Liddy C, Hogg W. Systematic review and meta-analysis of practice facilitation within primary care settings. Ann Fam Med 2012;10(1): 63-74.

13. Hogg W, Baskerville N, Nykiforuk C, Mallen D. Improved preventive care in family practices with outreach facilitation: understanding success and failure. J Health Serv Res Policy 2002;7(4):195201.

14. Nutting PA, Crabtree BF, Stewart EE, Miller WL, Palmer RF, Stange KC, Jaen CR. Effect of facilitation on practice outcomes in the national demonstration project model of the patient-centered medical home. Ann Fam Med 2010;8(Suppl 1):S33-44.

15. Dickinson WP, Dickinson LM, Nutting PA, et al. Practice facilitation to improve diabetes care in primary care: A report from the EPIC randomized clinical trial. Ann Fam Med 2014;12:8-16.

16. Cohen DJ, Balasubramanian BA, Davis $M$, et al. Understanding care integration from the ground up: five organizing constructs that shape integrated practices. J Am Board Fam Med 2015;28:S7-S20.

17. Hall J, Cohen DJ, Davis M, et al. Preparing the workforce for behavioral health and primary care integration. J Am Board Fam Med 2015;28:S41-S51.

18. Cohen DJ, Davis M, Balsubramanian BA, et al. Integrating behavioral health and primary care: consulting, coordinating and collaborating among professionals. J Am Board Fam Med 2015;28:S21-S31.

19. Gunn R, Davis M, Hall J, et al. Designing clinical space for the delivery of integrated behavioral health and primary care. J Am Board Fam Med 2015;28: S52-S62.

20. Davis MM, Balasubramanian BA, Fernald D, et al. Clinician staffing, scheduling, and engagement strategies among primary care practices. delivering integrated care. J Am Board Fam Med 2015;28:S32-S40.

21. Balasubramanian BA, Fernald D, Dickinson LM, et al. REACH of interventions integrating primary care and behavioral health. J Am Board Fam Med 2015;28:S73S85.

22. Cifuentes M, Davis M, Gunn R, Fernald D, Dickinson WP, Cohen D. Electronic health record challenges, workarounds, and solutions observed in practices integrating behavioral health and primary care. J Am Board Fam Med 2015;28:S63-S72. 